\title{
Conhecimento de acadêmicos de Odontologia sobre a prescrição antibiótica em tratamentos endodônticos
}

Knowledge of students of Dentistry on antibiotic prescription in endodontic treatments

Conocimiento de estudiantes de Odontología sobre la prescripción de antibióticos en tratamientos de endodoncia

Rachel Reinaldo ARNAUD ${ }^{1}$

Jéssica Bianca Risucci de França COSTA ${ }^{1}$

Thaynara Cavalcante Moreira ROMÃO ${ }^{1}$

Manuela Gouvêia Campêlo dos SANTOS ${ }^{2}$

Fábio Victor Dias SILVA ${ }^{1}$

Kauana da Silva ANDRADE ${ }^{1}$

Liandra Pamela de Lima SILVA ${ }^{1}$

${ }^{1}$ Departamento de Odontologia, Centro Universitário de João Pessoa, UNIPÊ, 58053-000 João Pessoa - PB, Brasil

${ }^{2}$ Departamento de Odontologia do UNIESP, Centro Universitário, João Pessoa - PB, Brasil

\section{Resumo}

Introdução: O uso de antibióticos na endodontia tem sido cada vez mais restrito e há uma preocupação quanto ao uso dessas drogas. Objetivo: Avaliar o conhecimento dos acadêmicos de Odontologia do Centro Universitário de João Pessoa - UNIPÊ, do sexto e décimo períodos, sobre a prescrição de antibióticos no tratamento endodôntico. Material e método: A amostra correspondeu a 105 participantes. O estudo apresentou uma abordagem descritiva e exploratória, utilizando um questionário. Os dados foram tabulados em uma plataforma do Microsoft Excel e analisados mediante estatística descritiva (valores de frequência absoluta e percentual). Resultados: Os estudantes relataram que esporadicamente apresentam dúvidas ao prescrever antibióticos sistêmicos. A maior parte dos alunos concluintes não soube identificar qual o tipo de receituário deve ser utilizado para prescrever e a validade da receita a partir da data de emissão. O medicamento de primeira escolha, indicado como coadjuvante ao tratamento endodôntico, foi descrito corretamente. Os participantes das duas turmas, não apresentaram certeza quanto aos casos que merecem a prescrição antimicrobiana e quais os pacientes de risco em que é preconizado a realização da profilaxia antibiótica. Conclusão: A maioria dos acadêmicos avaliados apresentaram conhecimento razoável evidenciando a necessidade contínua de atualização dos alunos quanto aos protocolos estipulados.

Descritores: Antibiótico; Prescrição; Odontologia; Endodontia.

\section{Abstract}

Introduction: The use of antibiotics in endodontics has been increasingly restricted and there is concern about the use of these drugs. Objective: The aim of this study was to evaluate the knowledge of the sixth and tenth semester dental students of the University Center of João Pessoa - UNIPÊ about the prescription of antibiotics in endodontic treatment. Material and method: The sample consisted of 105 participants and the study presented a descriptive and exploratory approach using a questionnaire. Data were tabulated on a Microsoft Excel platform and analyzed using descriptive statistics (absolute and percentage frequency values). Results: Students reported that they sporadically have doubts when prescribing systemic antibiotics. Most graduating students were unable to identify which type of prescription should be used for prescribing and the validity of the prescription from the date of issue. The drug of first choice, indicated as an adjuvant to endodontic treatment, was correctly described, as well as the correct interval between doses and penicillin substitutes in case of hypersensitivity. However, the participants in both groups were not sure about the cases that deserve the antimicrobial prescription and which patients are at risk for antibiotic prophylaxis. There was no statistically significant difference for the distribution of the students' responses, although the sixth period had the highest number of correct answers. Conclusion: Most of the evaluated students presented reasonable knowledge, evidencing the continuous need for updating students regarding the stipulated protocols.

Descriptors: Antibiotic; Prescription; Dentistry; Endodontics.

\section{Resumen}

Introducción: El uso de antibióticos en endodoncia se ha restringido cada vez más y existe preocupación por el uso de estos fármacos. Objetivo: Evaluar los conocimientos de los estudiantes de Odontología del Centro Universitario de João Pessoa UNIPÊ, del sexto y décimo período, sobre la prescripción de antibióticos en el tratamiento endodóntico. Material y método: La muestra correspondió a 105 participantes. El estudio presentó un enfoque descriptivo y exploratorio, utilizando un cuestionario. Los datos se tabularon en una plataforma Microsoft Excel y se analizaron mediante estadística descriptiva (valores absolutos y de frecuencia porcentual). Resultados: Los estudiantes informaron que esporádicamente tienen dudas a la hora de prescribir antibióticos sistémicos. La mayoría de los estudiantes graduados no pudieron identificar qué tipo de receta se debe usar para recetar y la validez de la receta a partir de la fecha de emisión. El fármaco de primera elección, indicado como complemento del tratamiento endodóntico, se describió correctamente. Los participantes de ambas clases no estaban seguros de los casos que merecen prescripción antimicrobiana y qué pacientes están en riesgo en los que se recomienda la profilaxis antibiótica. Conclusión: La mayoría de los académicos evaluados presentaron conocimientos razonables, evidenciando la necesidad continua de actualizar a los estudiantes en cuanto a los protocolos estipulados.

Descriptores: Antibiótico; Prescripción; Odontología; Endodoncia.

INTRODUÇÃO

A terapia endodôntica tem por objetivo a total eliminação ou a diminuição significativa de microrganismos patogênicos dos canais radiculares, por meio do preparo químicomecânico. Bactérias e seus subprodutos são considerados os principais agentes etiológicos das patologias pulpares e periapicais, e provocam a persistência da infecção, devido à falha do tratamento endodôntico e/ou à microinfiltração coronária, que permite a recontaminação microbiana dos canais radiculares ${ }^{1}$.

As infecções endodônticas são polimicrobianas, e predominadas por anaeróbios estritos gram-negativos, algumas praticamente assintomáticas e outras associadas a infecções graves. O uso de antibióticos como coadjuvante ao tratamento 
dessas infecções é uma importante alternativa quando clinicamente indicado. Contudo, a grande maioria das infecções de origem endodôntica é tratada sem a necessidade de emprego de antibióticos, pois estes não conseguem alcançar e eliminar microrganismos presentes no sistema de canais radiculares, devido à ausência de circulação sanguínea em polpas necrosadas ${ }^{1-6}$.

A escolha do antibiótico a ser prescrito, quando necessário, deve ser baseada em dados laboratoriais, saúde do paciente, idade, história de alergia, absorção, capacidade de distribuição do fármaco. Entretanto, em razão da demora de dias para a obtenção dos resultados de exames microbiológicos e de testes de suscetibilidade e diante da necessidade de prescrever um antibiótico imediatamente, a escolha deve ser baseada também no conhecimento atualizado do profissional sobre a microbiologia endodôntica ${ }^{7}$.

Diante do exposto, a otimização do conhecimento acerca do consumo de antimicrobianos na comunidade acadêmica, é de suma importância para proporcionar informações que racionalizem a utilização destes, contribuindo para que os futuros cirurgiões-dentistas analisem e saibam as reais necessidades e formas corretas de prescrição. Assim, este trabalho tem como objetivos, avaliar - conhecimento dos acadêmicos de Odontologia do Centro Universitário de João Pessoa (UNIPÊ), do sexto e décimo períodos, sobre a prescrição de antibióticos no tratamento endodôntico, e comparar os resultados de acordo com os períodos avaliados.

MATERIAL E MÉTODO

Trata-se de uma pesquisa exploratória quantitativa descritiva, realizada na Clínica Escola de Odontologia, com alunos do sexto e décimo períodos do UNIPE, no período de agosto de 2017. Foram atendidos os requisitos propostos pelo Conselho Nacional de Saúde/Ministério da Saúde, através da Resolução $n^{\circ} 466 / 2012^{4}$. A amostra foi composta por 151 alunos, de ambos gêneros, maiores de 18 anos, Após o cálculo amostral, a partir do universo de 151 alunos, com um nível de confiança de $95 \%$ e erro de $5 \%$, a amostra final foi de 105 alunos participantes. Como critério de inclusão da amostra na pesquisa foram analisadas informações como: estar matriculado $6^{\circ}$ ou no $10^{\circ}$ períodos do curso de graduação em Odontologia no UNIPÊ, ter sido aprovado nas disciplinas de Endodontia I, Endodontia II, Bioquímica, Farmacologia e Terapêutica, ser maior que 18 anos e concordar em participar da pesquisa assinando o Termo de Consentimento Livre e Esclarecido - TCLE. Foram excluídos desta pesquisa os acadêmicos que: sentiram-se constrangidos no preenchimento do instrumento de pesquisa e que preencheram o questionário de forma ilegível. Os acadêmicos tiveram o direito de abandonar a pesquisa a qualquer momento sem que houvesse nenhum prejuízo desta. Como instrumento de coleta de dados desta pesquisa foi utilizado um questionário estruturado com 9 questões objetivas. As informações obtidas com os questionários foram digitalizadas em uma planilha formada no programa EXCEL $\circledast 2013$ (Microsoft ${ }^{\circledR}$ Office ${ }^{\circledR}$ 2013), e analisados por meio de uma análise estatística descritiva e um tratamento estatístico percentual, por meio de dados absolutos e relativos.

RESULTADOS E DISCUSSÃO

A amostra correspondeu a 105 participantes, de um total de 151 acadêmicos, sendo 43 participantes do $6^{\circ}$ período e 62 do $10^{\circ}$ período, estando estes devidamente matriculados do curso de Odontologia do Centro Universitário de João Pessoa - UNIPÊ e obedecendo aos critérios de inclusão exigidos pela pesquisa.

A Tabela I mostra as distribuições absolutas e percentuais de acordo com perfil dos estudantes avaliadas. A maioria era do gênero feminino $(79,03 \%)$ e a faixa etária prevalente foi entre 21 e 30 anos, $85,48 \%$ do total da amostra, sendo a idade mínima de 18 anos e a máxima de 36 anos.

Tabela 1 - Distribuição absoluta e percentual dos estudantes de Odontologia do UNIPE do 6 ํ e $10^{\circ}$ períodos, segundo o gênero e faixa etária. João Pessoa - PB, Brasil - 2017.

\begin{tabular}{|c|c|c|c|c|}
\hline \multirow[t]{2}{*}{ SEXO } & \multicolumn{2}{|c|}{ 6 6 $^{\circ}$ PERÍODO } & \multicolumn{2}{|c|}{$10^{\circ}$ PERÍODO } \\
\hline & $N(43)$ & $\%$ & N (62) & $\%$ \\
\hline Feminino & 33 & $76,74 \%$ & 49 & $79,03 \%$ \\
\hline Masculino & 10 & $23,26 \%$ & 13 & $20,97 \%$ \\
\hline TOTAL & 43 & $100 \%$ & 62 & $100 \%$ \\
\hline \multirow[t]{2}{*}{ IDADE } & \multicolumn{2}{|c|}{$6^{\circ}$ PERÍODO } & \multicolumn{2}{|c|}{ 10' PERÍODO } \\
\hline & $N(43)$ & $\%$ & $N(62)$ & $\%$ \\
\hline 18-20 & 9 & $20,93 \%$ & 1 & $1,61 \%$ \\
\hline $21-30$ & 26 & $60,47 \%$ & 53 & $85,48 \%$ \\
\hline $31-40$ & 8 & $18,60 \%$ & 8 & $12,90 \%$ \\
\hline TOTAL & 43 & $100 \%$ & 62 & $100 \%$ \\
\hline
\end{tabular}

Inicialmente, foi questionado aos estudantes sobre o nível de segurança que sentem ao prescrever antibióticos sistêmicos. As opções variavam entre: A) Totalmente inseguro; B) Parcialmente inseguro; C) Frequentemente tenho dúvidas; D) Esporadicamente tenho dúvidas; E) Totalmente seguro. Na tabela II, pôde-se ver um percentual muito próximo entre as turmas quanto à segurança que sentem ao prescrever.

Os dados corroboram com a análise de Moura et $\mathrm{al}^{10}$. Que observou que $70 \%$ de um total de 500 participantes concordam que a disciplina de terapêutica não foi suficiente para garantir segurança para se prescrever 
medicamentos. Entretanto, devemos destacar que a falta de segurança e dúvidas podem estar relacionadas à falta de atualização contínua por parte dos estudantes. $O$ segundo questionamento consistia em saber se os alunos sabem identificar qual o tipo de receituário para prescrever antibióticos, e quantas vias são necessárias deste. Dentre as alternativas: A) Receituário comum (apenas 1 via), B) Receituário comum (2 vias), C). Apenas receituário de controle especial (2 vias), D). Apenas receituário de controle especial (1 via) + Notificação de receita tipo $B$ (cor azul), e letra E) Notificação de receita tipo $B$ (cor azul).

Os dados encontrados na Tabela 3 demonstram a falta de atualização do segundo grupo de participantes (10ำ período), bem como a explícita falta de conhecimento das normas para a prescrição de antibiótico; em contrapartida, grande parte dos alunos do $6^{\circ}$ período identificou corretamente a forma de prescrição desses fármacos, podendo isso estar associado com a proximidade desse período a disciplina de Terapêutica, que é ministrada no $4^{\circ}$ período. A elaboração de uma prescrição para medicamentos antimicrobianos é esclarecida pela RDC no 20 de 05 de maio de 2011, lançada nesse mesmo ano para revogar todas as outras resoluções anteriores sobre o tema. Esta orienta os dados obrigatórios que devem constar na receita, sendo essa em receituário simples, com retenção da $2^{\underline{a}}$ via pela farmácia e a $1^{\mathrm{a}}$ devolvida ao paciente ${ }^{4}$.

Tabela 2. Distribuição absoluta e percentual dos estudantes de odontologia do UNIPÊ do $6^{\circ}$ e $10^{\circ}$ períodos, segundo a segurança que julgam sentir ao prescrever medicamentos para o paciente. João Pessoa - PB, Brasil - 2017.

\begin{tabular}{|c|c|c|c|c|}
\hline & \multicolumn{2}{|c|}{$6^{\circ}$ PERÍODO } & \multicolumn{2}{|c|}{$10^{\circ}$ PERÍODO } \\
\hline & N (43) & $\%$ & $N(62)$ & \\
\hline $\mathbf{A}$ & 0 & $0 \%$ & 1 & $1,61 \%$ \\
\hline B & 7 & $16,28 \%$ & 8 & $12,90 \%$ \\
\hline $\mathbf{C}$ & 10 & $23,26 \%$ & 16 & $25,81 \%$ \\
\hline D & 23 & $53,49 \%$ & 31 & $50 \%$ \\
\hline $\mathbf{E}$ & 2 & $4,65 \%$ & 6 & $9,68 \%$ \\
\hline Não respondeu & 1 & $2,33 \%$ & & \\
\hline TOTAL & 43 & $100 \%$ & 62 & $100 \%$ \\
\hline
\end{tabular}

Tabela 3. Distribuição absoluta e percentual dos estudantes de odontologia do UNIPÉ do $6^{\circ}$ e $10^{\circ}$ períodos, segundo o tipo de receituário e número de vias que julgam necessárias para a prescrição de antibióticos. João Pessoa - PB - 2017.

\begin{tabular}{ccccc} 
& \multicolumn{2}{c}{$\mathbf{6}^{\mathbf{0}}$ PERÍODO } & \multicolumn{2}{c}{$\mathbf{1 0}^{\mathbf{0}}$ PERIODO } \\
\cline { 2 - 5 } & $\mathbf{N ( 4 3 )}$ & $\mathbf{\%}$ & $\mathbf{N}(\mathbf{6 2})$ & $\mathbf{\%}$ \\
\hline $\mathbf{A}$ & 0 & $0 \%$ & 1 & $1,61 \%$ \\
\hline $\mathbf{B}$ & 33 & $76,74 \%^{*}$ & 23 & $37,10 \%$ \\
\hline $\mathbf{C}$ & 8 & $18,60 \%$ & 36 & $58,06 \%$ \\
\hline $\mathbf{D}$ & 2 & $4,65 \%$ & 1 & $1,61 \%$ \\
\hline $\mathbf{E}$ & 0 & $\mathbf{0} \%$ & 1 & $1,66 \%$ \\
\hline TOTAL & $\mathbf{4 3}$ & $\mathbf{1 0 0} \%$ & $\mathbf{6 2}$ & $\mathbf{1 0 0} \%$ \\
\hline Fonte: Dados da pesquisa & & & \\
*Alternativa correta & & & &
\end{tabular}

$\mathrm{Na}$ Tabela 4, o quesito se referia à validade do receituário para antibióticos, que os alunos julgavam correta, em todo o território nacional. Dentre as opções estavam: A) 03 dias; B) 05 dias; C) 10 dias; D) 15 dias e E) 30 dias.
Tabela 4 - Distribuição absoluta e percentual dos estudantes de odontologia do UNIPÉ do $6^{\circ}$ e $10^{\circ}$ períodos, segundo a validade do receituário da prescrição antibiótica em território nacional. João Pessoa - PB, Brasil - 2017.

\begin{tabular}{|c|c|c|c|c|}
\hline & \multicolumn{2}{|c|}{ 60 PERÍODO } & \multicolumn{2}{|c|}{$1^{0^{\circ}}$ PERÍODO } \\
\hline & $\mathrm{N}(43)$ & $\%$ & $N(62)$ & $\%$ \\
\hline $\mathbf{A}$ & 0 & $0 \%$ & 1 & $1,61 \%$ \\
\hline B & 1 & $2,33 \%$ & 0 & $0 \%$ \\
\hline $\mathbf{C}$ & 29 & $67,44 \%^{*}$ & 24 & $38,71 \%$ \\
\hline D & 1 & $2,33 \%$ & 8 & $12,90 \%$ \\
\hline $\mathbf{E}$ & 12 & $27,91 \%$ & 29 & $46,77 \%$ \\
\hline TOTAL & 43 & $100 \%$ & 62 & $100 \%$ \\
\hline
\end{tabular}

Analisando os dados obtidos, para os discentes do $6^{\circ}$ período, a maioria $67,44 \%$ (29) marcou a opção adequada que dizia ser de 10 dias de validade a partir da data de emissão. Para os alunos que estão prestes a se formar, houve maior discordância entre eles, quando $46,77 \%(n=29)$ marcaram a opção de 30 dias de validade para o receituário com prescrição antibiótica, enquanto que apenas $38,71 \%$ $(\mathrm{n}=24)$ marcaram a opção adequada de 10 dias de validade.

Martins et al. ${ }^{9}$ realizaram um estudo relacionado aos erros ou omissão cometidos por prescritores, seja alunos ou profissionais. Foram avaliadas 228 receitas de medicamentos antibacterianos em Imperatriz - MA, onde 100\% das prescrições continham a identificação do prescritor, porém destas, apenas $87,7 \%$ tinham a data das prescrições, ficando assim impossibilitado de contabilizar a validade destas receitas e dispensar o medicamento.

A questão a qual a Tabela 5 expõe, perguntava aos participantes qual antibiótico de primeira escolha era prescrito, coadjuvante ao tratamento endodôntico, quando fosse necessário. As alternativas disponíveis eram: $\mathrm{A})$ Amoxicilina $500 \mathrm{mg}$, B) Clindamicina $300 \mathrm{mg}$, C) Metronidazol 250mg, D) Azitromicina 500mg, e E) Eritromicina $500 \mathrm{mg}$.

Tabela 5. Distribuição absoluta e percentual dos estudantes de odontologia do UNIPÊ do $6^{\circ}$ e $10^{\circ}$ períodos, segundo o antibiótico sistêmico de escolha prescrito para adultos saudáveis. João Pessoa - PB, Brasil - 2017

\begin{tabular}{|c|c|c|c|c|}
\hline & \multicolumn{2}{|c|}{$6^{\circ}$ PERÍODO } & \multicolumn{2}{|c|}{ 10\% PERÍODO } \\
\hline & $N(43)$ & $\%$ & N (62) & $\%$ \\
\hline $\mathbf{A}$ & 43 & $100 \% *$ & 60 & $96,77 \% *$ \\
\hline B & 0 & $\%$ & 1 & $1,61 \%$ \\
\hline $\mathbf{C}$ & 0 & $\%$ & o & $\mathrm{o} \%$ \\
\hline $\mathbf{D}$ & 0 & $\%$ & 1 & $1,61 \%$ \\
\hline $\mathbf{E}$ & 0 & $\%$ & 0 & $0 \%$ \\
\hline TOTAL & 43 & $100 \%$ & 62 & $100 \%$ \\
\hline
\end{tabular}

$\mathrm{Na}$ odontologia, a literatura é unânime quando afirma que o antibiótico de primeira escolha é a amoxicilina ${ }^{1,5}$, resultado esse que foi predominante na presente pesquisa, quando $100 \%$ dos alunos do $6^{\circ}$ período e a maioria do $10^{\circ}$ período marcaram este antimicrobiano. A questão 5, demonstrada na tabela abaixo, perguntava o intervalo entre doses que estes alunos costumavam prescrever o antibiótico de primeira escolha (Amoxicilina $500 \mathrm{mg}$ ) no 
tratamento de afecções endodônticas. As alternativas eram: A) De 4 em 4 horas, B) De 6 em 6 horas, C) De 8 em 8 horas, D) De 12 em 12 horas, e E) Apenas 1 vez ao dia.

Tabela 6. Distribuição absoluta e percentual dos estudantes de odontologia do UNIPE do 60 e $10^{\circ}$ períodos, segundo o intervalo correto entre doses do antibiótico de eleição para adultos saudáveis. João Pessoa - PB, Brasil - 2017.

\begin{tabular}{|c|c|c|c|c|}
\hline & \multicolumn{2}{|c|}{$6^{\circ}$ PERÍODO } & \multicolumn{2}{|c|}{$10^{\circ}$ PERÍODO } \\
\hline & (43) & $\%$ & $N(62)$ & $\%$ \\
\hline $\mathbf{A}$ & 0 & $0 \%$ & 0 & 0\% \\
\hline B & 5 & $11,63 \%$ & 3 & $4,84 \%$ \\
\hline C & 27 & $62,79 \%^{*}$ & 49 & $79,03 \%^{*}$ \\
\hline D & 11 & $25,58 \%$ & 8 & $12,90 \%$ \\
\hline $\mathbf{E}$ & o & $0 \%$ & 2 & $3,23 \%$ \\
\hline TOTAL & 43 & $100 \%$ & 62 & $100 \%$ \\
\hline
\end{tabular}

Houve concordância entre as maiorias das turmas, quando o percentual de alunos do $6^{\circ}$ e $10^{\circ}$ períodos, que escolheu a opção de prescrever a amoxicilina "de 08 em 08 horas", correspondeu à mais da metade, apesar de que poucos alunos ainda optaram pela escolha de duas vezes ao dia. Isto pode ser justificado pela confusão que ainda é feita quanto às doses prescritas para diferentes antimicrobianos em conjunto com as doses estabelecidas para os outros grupos de medicamentos.

Pode-se observar consonância à pesquisa de Lucchette et al. ${ }^{8}$, quando observou que cirurgiões-dentistas da rede pública de um município de São Paulo, quando perguntados quanto ao antibiótico mais prescrito, dosagem e intervalo entre doses, $97,3 \%$ de um total de 74 , citaram a amoxicilina, por via oral, na dosagem de $500 \mathrm{mg}$. Os valores oscilaram quanto ao intervalo entre doses, pois $87,8 \%$ prescreveriam a cada 8 horas, $2,7 \%$ a cada 12 horas e $9,5 \%$ a cada 6 horas.

A Tabela 7 mostra as opções escolhidas pelos participantes, quanto aos antibióticos coadjuvantes ao tratamento endodôntico que poderiam ser utilizados como substitutos às penicilinas em caso de hipersensibilidade a esse grupo de fármacos. As opções consistiam em: A) Amoxicilina (500mg); B) Clindamicina (300mg); C) Cefalexina (500mg); D) Azitromicina (500mg) e E) Eritromicina (500mg). Este quesito aceitou que os alunos assinalassem mais de uma opção, dependendo da interpretação individual.

Para Lopes e Siqueira Júnior ${ }^{6}$, pacientes com histórico de hipersensibilidade às penicilinas, a clindamicina na dosagem de $300 \mathrm{mg}$ de 6 (seis) em 6 (seis) horas parece ser a melhor opção. Sendo assim, os resultados obtidos nessa pesquisa se mostraram favoráveis, quando constatado que nenhum dos alunos escolheu a opção de amoxicilina, expondo que conheciam o grupo à qual pertence tal medicamento (penicilinas), evitando assim a administração inadequada deste.
Tabela 7. Distribuição absoluta e percentual dos estudantes de odontologia do UNIPÉ do $6^{\circ}$ e $10^{\circ}$ períodos, segundo os antibióticos sistêmicos prescritos aos pacientes em caso de alergia à penicilina. João Pessoa - PB, Brasil - 2017.

\begin{tabular}{|c|c|c|c|c|}
\hline & \multicolumn{2}{|c|}{$6^{\circ}$ PERÍODO } & \multicolumn{2}{|c|}{$10^{\circ}$ PERÍODO } \\
\hline & N (43) & $\%$ & N (62) & $\%$ \\
\hline $\mathbf{A}$ & 0 & $0 \%$ & 0 & $0 \%$ \\
\hline B & 35 & $79,55 \%$ & 56 & $81,16 \%$ \\
\hline $\mathbf{C}$ & 0 & $0 \%$ & 2 & $2,90 \%$ \\
\hline D & 8 & $18,18 \%$ & 7 & $19,14 \%$ \\
\hline $\mathbf{E}$ & 1 & $2,27 \%$ & 4 & $5,80 \%$ \\
\hline TOTAL & $44^{*}$ & $100 \%$ & $69^{*}$ & $100 \%$ \\
\hline
\end{tabular}

A Tabela 8 mostra os resultados referentes à abordagem quanto às afecções endodônticas que se faz necessária a prescrição antibiótica. Dentre as alternativas: A) Pulpite reversível, B) Pulpite Irreversível, C) Dor relacionada a traumatismo, D) Edema sem complicações induzido por traumatismo ou substâncias químicas, E) Bactérias limitadas ao canal radicular, F) Necrose pulpar sem envolvimento periapical e sem sintomatologia de disseminação infecciosa, G) Necrose pulpar com envolvimento periapical e sem sintomatologia de disseminação infecciosa, $H$ ) Periodontite apical aguda, I) Periodontite apical crônica, J) Abscesso perirradicular agudo com ocorrência de tumefação difusa e/ou envolvimento sistêmico, K) Abscesso perirradicular agudo com tumefação localizada e sem envolvimento sistêmico, L) Abscesso perirradicular crônico, M) Trato fistuloso (presença de fístula) de drenagem crônica, N) Abscesso fênix (flare-ups), O) Avulsão dentária e P) Sintomatologia e exsudação persistente.

Tabela 8. Distribuição absoluta e percentual dos estudantes de odontologia do UNIPÊ do $6^{\circ}$ e $10^{\circ}$ períodos, segundo as afecções endodônticas que necessitam de prescrição antibiótica. João Pessoa - PB, Brasil - 2017.

\begin{tabular}{|c|c|c|c|c|}
\hline & \multicolumn{2}{|c|}{$6^{\circ}$ PERÍODO } & \multicolumn{2}{|c|}{$10^{\circ}$ PERÍODO } \\
\hline & N (43) & $\%$ & N (62) & $\%$ \\
\hline $\mathbf{A}$ & 1 & $0,61 \%$ & 4 & $1,54 \%$ \\
\hline $\mathbf{B}$ & 2 & $1,22 \%$ & o & $0 \%$ \\
\hline $\mathbf{C}$ & 2 & $1,22 \%$ & 1 & $0,38 \%$ \\
\hline D & 1 & $0,61 \%$ & 6 & $2,31 \%$ \\
\hline $\mathbf{E}$ & 10 & $6,10 \%$ & 10 & $3,85 \%$ \\
\hline $\mathbf{F}$ & 2 & $1,22 \%$ & 0 & $0 \%$ \\
\hline $\mathbf{G}$ & 4 & $2,44 \%$ & 10 & $3,85 \%$ \\
\hline $\mathbf{H}$ & 12 & $7,32 \%$ & 17 & $6,54 \%$ \\
\hline I & 11 & $6,71 \%$ & 4 & $1,54 \%$ \\
\hline $\mathbf{J}$ & 39 & $23,78 \% *$ & 55 & $21,15 \%$ \\
\hline $\mathbf{K}$ & 4 & $2,44 \%$ & 29 & $11,15 \%$ \\
\hline $\mathbf{L}$ & 12 & $7,32 \%$ & 29 & $11,15 \%$ \\
\hline $\mathbf{M}$ & 22 & $12,41 \%$ & 27 & $10,38 \%$ \\
\hline $\mathbf{N}$ & 16 & $9,76 \% *$ & 20 & $7,69 \% *$ \\
\hline $\mathbf{O}$ & 2 & $1,22 \%{ }^{*}$ & 16 & $6,15 \% *$ \\
\hline $\mathbf{P}$ & 24 & $14,63 \%^{*}$ & 32 & $12,31 \%^{*}$ \\
\hline TOTAL & $164^{* * *}$ & $100 \%$ & $260^{* * *}$ & $100 \%$ \\
\hline
\end{tabular}

Com relação às afecções endodônticas que os alunos do 6ำ período julgam necessitar de prescrição antibiótica, no geral, estes prescreveriam para todas as situações apresentadas na questão, ou seja, todas os casos expostos possuíram pelo menos um voto, mostrando que os alunos ainda se mostram indecisos quanto a real necessidade. Os participantes do $10^{\circ}$ período também não apresentaram certeza quanto aos casos que 
merecem a prescrição antimicrobiana. Nos resultados, notamos antibióticos sendo prescritos independente dos casos e fases evolutivas, para abscessos, pulpites, dor por traumatismos, e a maioria destes sem sintomatologia de disseminação infecciosa, casos esses que a literatura não aponta como uma real necessidade, a não ser que haja comprometimento sistêmico[10]. É importante destacar que o uso incorreto destes agentes terapêuticos pode levar ao surgimento de microrganismos resistentes, no entanto, os antibióticos são frequentemente prescritos pelos cirurgiões-dentistas no tratamento destas situações ${ }^{11}$. Lopes e Siqueira Júnior ${ }^{6}$, afirmam que em ocorrências que exigem drenagem de abscessos e envolvimento sistêmico do paciente, ou que o sistema imunológico não sirva de barreira para a disseminação da infecção, mesmo se a drenagem foi lograda satisfatoriamente, deve-se empregar antibióticos. Foi perguntado ainda em quais casos de pacientes de risco é preconizado a realização da profilaxia antibiótica no tratamento endodôntico. Dentre as opções estavam: A) Prevenção de endocardite infecciosa em pacientes suscetíveis; B) Gestantes; C) Diabéticos compensados; D) Diabéticos descompensados; E) Hipertensos; F) Imunodeprimidos; G) Pacientes com próteses ortopédicas; H) Pacientes recebendo quimioterapia (leucócitos <2.500); I) Transplantes de medula óssea (leucócitos $<2.500)$; J) Pacientes com marcapasso (Tabela 9).

Tabela 9. Distribuição absoluta e percentual dos estudantes de odontologia do UNIPÊ do $6^{\circ}$ e $10^{\circ}$ períodos, segundo os casos de pacientes que necessitam de profilaxia antibiótica no tratamento endodôntico. João Pessoa - PB, Brasil - 2017.

\begin{tabular}{|c|c|c|c|c|}
\hline & \multicolumn{2}{|c|}{$6^{\circ}$ PERÍODO } & \multicolumn{2}{|c|}{$10^{\circ}$ PERÍ́DO } \\
\hline & N (43) & $\%$ & $N(62)$ & $\%$ \\
\hline $\mathbf{A}$ & 40 & $34,48 \%^{*}$ & 57 & $26,89 \%^{*}$ \\
\hline B & 1 & $0,86 \%$ & 0 & \\
\hline C & 1 & $0,83 \%$ & 3 & $1,42 \%$ \\
\hline D & 16 & $13,79 \%^{*}$ & 23 & $10,85 \%^{*}$ \\
\hline $\mathbf{E}$ & 0 & o\% & 4 & $1,89 \%$ \\
\hline $\mathbf{F}$ & 14 & $12,07 \%{ }^{*}$ & 34 & $16,04 \% *$ \\
\hline G & 7 & $6,03 \% *$ & 20 & $9,43 \%^{*}$ \\
\hline $\mathbf{H}$ & 10 & $8,62 \%^{*}$ & 15 & $7,08 \% *$ \\
\hline I & 10 & $8,62 \%^{*}$ & 19 & $8,96 \% *$ \\
\hline $\mathbf{J}$ & 17 & $14,66 \%$ & 37 & $17,45 \%$ \\
\hline TOTAL & $116^{* * *}$ & $100 \%$ & $212^{* * *}$ & $100 \%$ \\
\hline
\end{tabular}

Ambos períodos tiveram seu maior percentual de votos ao afirmarem prescrever antibioticoterapia profilática para prevenir a endocardite infecciosa em pacientes suscetíveis. Trento et al. ${ }^{11}$, na sua pesquisa envolvendo 80 alunos e 50 profissionais cirurgiões dentistas da cidade Aracajú-SE verificou que, quando perguntados sobre e situação em que seria indicada a profilaxia antibiótica, $87,5 \%$ dos acadêmicos e $86 \%$ dos profissionais concordaram com a opção de pacientes com risco de endocardite bacteriana. Entretanto, estes participantes também votaram nas situações de "qualquer procedimento invasivo" $(6,3 \%$ e $18 \%)$, hipertensos $(6,3 \%$ e $2 \%)$ e em endodontias $(8,8 \%$ e $6 \%)$, evidenciando assim a falta da busca pela informação e estudo desde a graduação, dando continuidade a esse quadro, quando então profissionais.

A outra opção explorada, é quanto aos pacientes diabéticos descompensados e os imunodeprimidos que incluem pacientes transplantados e que estão recebendo quimioterapia, ambos com leucopenia $(<2.500)$, os quais merecem atenção quanto à necessidade de profilaxia ${ }^{8}$.

A tabela 10 descreve os resultados referentes ao questionamento da melhor opção de dose de ataque de profilaxia antibiótica recomendada pela American Heart Assotiation (AHA). Dentre as opções estavam: A) Amoxicilina de $(500 \mathrm{mg})$, sendo $1 \mathrm{~g}$; B) Amoxicilina (500mg), sendo 2g; C) Clindamicina (300mg), sendo 600mg; D) Azitromicina (500mg), sendo $1 \mathrm{~g}$ via oral; e E) Cefalexina (500mg), sendo $2 \mathrm{~g}$ também por via oral, todos 1 hora antes do procedimento.

Tabela 10. Distribuição absoluta e percentual dos estudantes de odontologia do UNIPÉ do $6^{\circ}$ e $10^{\circ}$ períodos, segundo 0 medicamento de escolha para realizar profilaxia antibiótica em pacientes não alérgicos à penicilina. João Pessoa - PB, Brasil 2017.

\begin{tabular}{|c|c|c|c|c|}
\hline & \multicolumn{2}{|c|}{$6^{\circ}$ PERÍODO } & \multicolumn{2}{|c|}{$10^{\circ}$ PERÍODO } \\
\hline & N (43) & $\%$ & N (62) & $\%$ \\
\hline $\mathbf{A}$ & 5 & $11,63 \%$ & 16 & $25,81 \%$ \\
\hline B & 35 & $81,40 \%{ }^{*}$ & 43 & $69,35 \% *$ \\
\hline $\mathbf{C}$ & 1 & $2,33 \%$ & 2 & $3,23 \%$ \\
\hline D & $\mathrm{o}$ & $0 \%$ & $\mathrm{o}$ & $0 \%$ \\
\hline $\mathbf{E}$ & 0 & $0 \%$ & 1 & $1,61 \%$ \\
\hline Não Respondeu & 2 & $4,65 \%$ & - & - \\
\hline TOTAL & 43 & $100 \%$ & 62 & $100 \%$ \\
\hline
\end{tabular}

Nessa etapa, a maioria dos alunos de ambas turmas marcaram a alternativa que continha a Amoxicilina como antibiótico de escolha para pacientes não alérgicos, na concentração de $500 \mathrm{mg}$, sendo a posologia de $2 \mathrm{~g}$ por via oral em dose única, 01 hora antes do procedimento, corroborando com a pesquisa de Trento et al. ${ }^{11}$, na qual $100 \% \quad(n=50)$ dos profissionais entrevistados, prescreviam Amoxicilina como primeira opção para a profilaxia antibiótica, seguido da azitromicina (36\%).

Diversos autores também confirmam o uso profilático da Amoxicilina em dose única, $2 \mathrm{~g}, 1$ hora antes do procedimento, como antibiótico de primeira escolha, protocolo esse preconizado pela AHA (American Heart Association) em 2007, por ser melhor absorvido no trato gastrointestinal e por proporcionar 
níveis séricos mais elevados e duradouros, além da sua eficácia contra microrganismos causadores da infecção $0^{5,12}$.

Em vista do que foi explorado nesta pesquisa, esta mostra o conhecimento razoável dos estudantes em relação aos casos que necessitam da prescrição de antibióticos, revelando um problema na formação acadêmica destes, e evidenciando a necessidade contínua de atualização dos alunos quanto aos protocolos estipulados e pelas literaturas vigentes na escola, de forma a diminuir a insegurança e as consequências decorrentes do uso incorreto dos antibióticos.

CONCLUSÃO

De acordo com os resultados obtidos nesse trabalho, pode-se concluir que a maioria dos estudantes relatou que esporadicamente apresentam dúvidas ao prescrever antibióticos sistêmicos no tratamento endodôntico. A maior parte dos alunos concluintes não souberam identificar qual o tipo de receituário deve ser utilizado para prescrever antibióticos e a validade da receita a partir da data de emissão, diferenciando dos resultados obtidos pelos alunos do $6^{\circ}$ período. O medicamento de primeira escolha, indicado como coadjuvante ao tratamento endodôntico, foi descrito corretamente por grande parte dos alunos, assim como, o intervalo correto entre doses e os substitutos às penicilinas em caso de hipersensibilidade a esse grupo de fármacos. Os participantes das duas turmas, não apresentaram certeza quanto aos casos que merecem a prescrição antimicrobiana no tratamento endodôntico. Não houve diferença estatisticamente significante, na maioria das variáveis abordadas, para a distribuição das respostas dos alunos avaliados, dos diferentes períodos do curso, embora o sexto período tenha apresentado o maior número de acertos quando comparados ao décimo.

\section{REFERÊNCIAS}

1. Di Santi BT, Ribeiro BM, Endo MS, Gomes BFA. Avaliação da suscetibilidade antimicrobiana de bactérias anaeróbias facultativas isoladas de canais radiculares de dentes com insucesso endodôntico frente aos antibióticos de uso sistêmico. Rev Odontol UNESP. 2015;44(4):200-6.

2. Alfenas CF, Lins FF, Maneschy MT, De Uzesa M. Antibióticos no tratamento de abscessos perirradiculares agudos. Rev bras odontol. 2014;71(2):120-3.

3. Andrade E. Terapêutica Medicamentosa em Odontologia: da excelência à regulação das aprendizagens entre duas lógicas. $3^{\circ}$ ed. Rio de Janeiro: Artes Médicas; 2014. p. 119-4.
4. Brasil. Ministério da Saúde. Agência Nacional de Vigilância Sanitária. Resolução da Diretoria Colegiada RDC no 20 de 5 de maio de 2011. DO 44 de 9/05/2011.

5. De Andrade SLC, Faria JMP, Andrade JDS, Souza LG, Fernandes AB, Botelho $M$, et al. Avaliação da antiboticoterapia na Odontologia. Arq Bras Odontol. 2017;11(2):1-6.

6. Lopes HP, Siqueira Junior JF. Endodontia: biologia e técnica. Rio de Janeiro: Elsevier Brasil; 2015.

7. Sousa EL, Torino GG, Martins GB. Antibióticos em Endodontia: por que, como e quando usálos. São Paulo: Santos; 2014.

8. Lucchette, ACT, Tenani CF, Possobon RF, Batista MJ. Avaliação da prática de prescrição de antibióticos pelos cirurgiões-dentistas da rede pública do município de médio porte. Arq Odontol. 2019;55(6):1-11.

9. Martins NB, De Sousa LMG, Torres MLD, Firmo WCA. Análise De Prescrição Médica De Antibióticos De Uma Farmácia Comercial Do Município De Imperatriz-Ma. Rev Cient ITPAC. 2017;7(4):1-9.

10. Moura, CS; Naves, JOS; Coelho, EB; Lia, EN. Avaliação da qualidade da prescrição por estudantes de Odontologia. J Appl Sci Oral, Bauru. 2014;22(3):204-8.

11. Trento CL, Júnior LRM, Siqueira ADS, Takeshita WM. Avaliação do conhecimento de Cirurgiões-Dentistas e acadêmicos de Odontologia na cidade de Aracaju, Sergipe, a respeito da adequada prescrição de antimicrobianos. Rev Odontol UNESP. 2014; 43(4):286-93.

12. Bernardo JE, Loureiro $\mathrm{C}$, Jacinto RC. Análise da resistência de bactérias isoladas de infecções endodônticas a antibióticos: uma revisão de literatura. Arch Health Invest. 2018;7(Spec Iss 3):51.

\section{CONFLITO DE INTERESSES}

Os autores declaram não haver conflitos de interesse

AUTOR PARA CORRESPONDÊNCIA

\section{Thaynara Cavalcante Moreira Romão}

Rua Jorge Faraj, 71 - Miramar,

58032-010 João Pessoa - PB, Brasil

E-mail: thaynaracavalcante1@gmail.com 